\title{
MALGRÉ L'HÉRITAGE : DE CE QUI RESTE EN SUSPENS ENTRE FREUD ET DERRIDA
}

\section{DESPITE THE INHERITANCE: ABOUT THAT WHICH IS PENDING BETWEEN FREUD AND DERRIDA}

\author{
JAVIER AgüERo ÁGUILA* \\ Université Paris 8 Vincennes-Saint-Denis
}

RÉsumé : Ce travail présente une scène virtuelle de l'héritage entre Freud et Derrida. Héritage compris ici non pas comme ce qui se déplace depuis celui qui hérite jusqu’au légataire sous une forme passive et sans objection, mais au contraire comme un héritage assumé en tant qu’altération de ce dont on hérite. À partir de cette idée philosophique de l'héritage que défend Derrida, celui-ci nous serait révélé resignifié, a-temporel et en constante transformation. Néanmoins, il sera important de ne pas condenser une scène probable de l'héritage entre Freud et Derrida en une sorte de dispute ou d'équivalence entre les concepts spécifiques de la psychanalyse et ceux de la déconstruction. Nous nous demandons quel est le nom propre de la psychanalyse et de quoi nous parlons quand nous parlons de Freud et de son impact dans l'œuvre derridienne. Nous nous posons, finalement, cette question : qu'est-ce que Derrida choisit et altère en tant qu'héritage? Freud ou la psychanalyse?

Mots Clés : Derrida, Freud, Héritage, Principe du plaisir

Sociologue, Master en Philosophie Politique à l'Université du Chili. Doctorant en philosophie à l'Université Paris 8 Vincennes / Saint-Denis. Membre du Laboratoire d'Études et de Recherches sur les Logiques Contemporaines de la Philosophie - LLCP/ Université Paris 8. 
АвSTRACT: This article proposes a potential scene of the inheritance between Freud and Derrida. Inheritance is understood here not as that which is passed from one legatee to an heir in a passive way and with no objections; rather, on the contrary, inheritance is assumed to be an alteration of that which is inherited. From this philosophical idea of inheritance, defended by Derrida, this idea reveals itself to us as a re-significant, timeless one that is in constant transformation. Nevertheless, as will be seen, it is important not to summarize a probable scene of the inheritance between Freud and Derrida in a sort of dispute or equivalence between the specific concepts of psychoanalysis and deconstruction. The question I ask is: What is the proper name of Freudian heritage beyond the institution of psychoanalysis and what are we talking about when we speak about Freud and his impact on Derrida's work? Finally, I wonder what is chosen and altered as the inheritance by Derrida? Freud or psychoanalysis?

KeYwords: Derrida, Freud, Inheritance, Pleasure principle

À Carlos Contreras, l'ami dans la question

"Je ressemble à un messager de l'antiquité, un coursier, le courrier de ce que nous nous sommes donné, à peine un héritier, un héritier infirme, incapable de recevoir même, de se mesurer à ce dont il a la garde ». J. Derrida

\section{Introduction}

Tenter d'élaborer un texte sur l'héritage possible - ou resté en suspend entre Freud et Derrida représente dès le départ, entre autres, une difficulté liée à la définition de cette notion et au point à partir duquel il faudra partir. L'héritage n'est pas, en termes de rigueur linguistique, un concept philosophique et sa définition nous indique plusieurs possibilités interprétatives qui l'éloigneraient d'un examen proprement philosophique. 
Selon le Dictionnaire de la langue française Le Littré, l'héritage peut être compris, au moins, de trois façons. En premier lieu comme « ce qui vient par voie de succession " (une culture, un trône ou un royaume, par exemple) ; une deuxième définition concerne des éléments plus particuliers tels que "les immeubles réels, terres, maisons, etc. $»^{1}$. Dans les deux cas, il s'agirait d'un transfert de biens symboliques ou matériels qui se déplacent vers quelqu'un ou vers quelque chose. En considérant ces deux définitions, on se trouve alors face à un concept qui, à partir de son explication la plus originale, ne facilite pas son traitement philosophique ou, pour le moins, celui-ci ne résulte pas spontané ni facile non plus suivre sa trace au sein de la philosophie elle-même.

Cependant, si l'on insiste, l'on se trouve face à une troisième définition de l'héritage qui, sans vouloir l'être ou plutôt par hasard, aurait tendance à indiquer la voie pour une analyse philosophique de l'héritage, à savoir : " Ce qui arrive, comme arrive un héritage $»^{2}$.

L'involontaire puissance philosophique de cette définition se révèle à nous immédiatement comme une clé, un code d'accès pour précisément repenser l'intuition derridienne de ce que pourrait être l'héritage. Ceci nous laisse penser que l'héritage est toujours en suspens - en train d'arriver-. S'il ne se produit pas encore, il promet de le faire. Cependant, au moment où il se produit, l'héritage cesse d'être un droit sans nom propre et en arrive à avoir force de loi, vigueur historique, identité. Je n'ai le droit sur quelque chose qu'au moment où ce quelque chose m'appartient. Mais nous voulons penser l'héritage comme quelque chose en état d'envoi permanent. C'est La carte postale derridienne qui est expédiée sans adresse et sans expéditeur, toujours en route et résistante à toute destination. C'est le trafic d'héritage (Derrida, 1980, 393-412) qui irait de Socrate à Freud sans se laisser consumer en un quelconque schéma d'appropriation spécifique et définitive. Trafic d'héritage dont, bien sûr, Derrida sera toujours un héritier infidèle en attente. On reviendra plus loin sur ce thème.

\footnotetext{
${ }^{1}$ Voir : http://www.littre.org/definition/h\%C3\%A9ritage

2 Ibid
} 
Avant même de proposer toute scène d'héritage entre la psychanalyse freudienne et la déconstruction derridienne, il sera alors nécessaire d'approfondir ce que nous entendons lorsque nous pensons l'héritage à partir de Jacques Derrida.

\section{Héritage et double jeu : l'amour des infidèles}

Nous commencerons par cette citation de Derrida :

(...) la déconstruction la plus rigoureuse ne s'est jamais présentée (...) comme quelque chose de possible. Je dirais qu' elle ne perd rien à s'avouer impossible, et ceux qui s'en réjouiraient trop vite ne perdent rien pour attendre. Le danger pour une tâche de déconstruction, ce serait plutôt la possibilité, et de devenir un ensemble disponible de procédures réglées, de pratiques méthodiques, de chemins accessibles. (Derrida, 1987, 26-27).

Nous citons ce texte pour signaler dès à présent que l'héritage est, au sens philosophique, comme le souligne Derrida au sujet de la déconstruction, une expérience aporétique de l'impossible. Par-là, nous voulons dire que l'héritage compris comme un legs coordonné du début à la fin ou, plutôt, comme un corpus qui se déplacerait d'un point $\mathrm{X}$ à un autre point $\mathrm{Y}$ (de façon spécifique, celui qui hérite et celui qui est le légataire) sans faire de détour et sans être altéré au cours de ce déplacement, cet héritage résulte être, du point de vue de la pensée derridienne, une expérience impossible. Certes, nous n'essaierons pas de faire un simple exercice d'algèbre de rotation des concepts entre la déconstruction et l'héritage, mais plutôt de nous attacher au fait de penser que l'héritage même ne peut être compris que dans l'évènement d'une figure impossible ${ }^{3}$. C'est en ce sens que la déconstruction apparaîtrait, au-delà de toute équivalence entre les concepts, comme une scène d'héritage.

C'est peut-être pour cette raison que Derrida note ceci dans Points de suspension: "je me sens aussi héritier : fidèle autant que possible " (Derrida,

3 Il faut préciser que, pour Derrida, l'impossible n'est pas le contraire du possible, il n'y a pas de contradiction logique entre ces deux dimensions. Ce qui se passerait est que l'impossible se révèle comme l'unique condition de possibilité du possible ou, encore, c'est seulement dans l'orbite de l'impossible que le possible laisse entrevoir quelque horizon d'actualisation, bien que cela même soit impossible. À ce sujet, voir par exemple : Derrida. J., Papier Machine, Galilée, Paris, 2001, p. 303. cf. aussi : De Peretti, C., «Herencias de Derrida ", in Isegoría n³2, Madrid, 2005, p. 122 
1998, 139). Fidèle autant que possible. Cette phrase à elle-même semblerait ne pas faire l'économie sur ce qui arriverait à représenter une idée de l'héritage dans l'œuvre derridienne, mais plutôt alerter à son sujet. Comme cela a été signalé, si l'héritage est une expérience de l'impossible, tout en sachant que c'est cependant là (et non ailleurs) qu'il pourrait avoir quelque possibilité, la phrase fidèle autant que possible condense ce que l'on pourrait dénommer une eidétique de l'héritage. Nous insistons, fidèle autant que possible renvoie à quelque chose qui ne sera jamais possible, mais qui est toujours en train de l'être. Selon les propres termes de Jacques Derrida :

Quand l'impossible se fait possible, l'événement a lieu (possibilité de l'impossible). C'est même là, irrécusablement, la forme paradoxale de l'événement (...). Pour qu'un événement ait lieu, pour qu'il soit possible, il faut qu'il soit, comme événement, comme invention, la venue de l'impossible " (Derrida, 2001, 307).

Comment comprendre alors Derrida comme un héritier potentiel de Freud si l'héritage est toujours à-venir et jamais une actualisation de lui-même ? Nous essaierons de commencer par cette réponse de Derrida lui-même : " je me vois souvent passer très vite devant le miroir de la vie, comme la silhouette d'un fou (à la fois comique et tragique) qui se tue à être infidèle par esprit de fidélité " (Derrida ; Roudinesco, 2001, 14-15). L'infidélité, comme nous le verrons, est l'horizon possible de l'héritage à l'intérieur de sa condition impossible. C'est le geste infidèle qui le conduirait à être une trace possible, un trait, une cendre ou, en dernier ressort, une différance (Derrida, 1968), (celle-ci comprise comme dilation et contexte, espacement et corps. Différance adhérée à toute manifestation exprimée par et à partir de l'héritage en ce cas) au centre de l'assimilation du légataire. L'héritier est loin d'être cette figure commode et sédentaire en attente de l'héritage comme s’il était un envoi dirigé et expédié. Il y a, dans cette perspective, une double injonction, à laquelle l'héritier devrait répondre ${ }^{4}$. En premier lieu, il est nécessaire de "savoir réaffirmer ce qui vient " avant nous" , et que donc nous recevons avant même de le choisir, et de nous comporter à cet

${ }^{4}$ Rappelons que pour Derrida l'« injonction " est le moment temporel et dilatoire qui regroupe deux instants mais qu'il n'est pas capable d'unir. À savoir que bien qu'imbriqués, ils sont de nature parallèle. C'est en même temps le moment du non planifié et de l'imminent, ainsi que l'espace radical et possible pour l'immanence de l'évènement. cf. par exemple : Derrida, J., Spectres de Marx. L'État de la dette, le travail du deuil et la nouvelle International, Galilée, Paris, 1993, p. 269 
égard en sujet libre» (Derrida ; Roudinesco, 2001, 15). L'héritier passe alors par l'expérience d'assumer ce qui préexiste sans savoir précisément de quoi il s'agit. Nous ne choisissons pas nous-mêmes notre héritage, c'est lui qui nous choisit parce que nous essayons, à la suite de Derrida, de nous approprier d'un passé que nous savons inapropriable, étranger à toute formalisation ou actualisation présente et dont finalement, nous méconnaissons la condition de plein devenir. Cependant, l'héritage doit être réaffirmé.

"Réaffirmer, qu'est-ce que ça vaut dire ? Non seulement l'accepter, cet héritage, mais le relancer autrement et le maintenir en vie " (Derrida; Roudinesco, 2001, 15). La réaffirmation de l'héritage dans ces lignes est une réaffirmation de cet inconnu qui nous a choisi et auquel nous devons alors donner cours, donner vie. Réaffirmation et réactivation d'un legs vis-à-vis duquel nous sommes infidèles par principe de fidélité et donc par principe de responsabilité. Il ne serait pas possible d'étendre un héritage ni de lui rendre « justice » si ce n'est en l'altérant, en le rendant hétérogène et irréductible à toute confirmation d'un présent performatif et temporel.

Cependant, et c'est là la seconde injonction, cette réception qui est en même temps don exige de la part de celui qui reçoit l'héritage une sélection, un filtrage ou une décision en face de la possibilité infinie de ce qui est offert. Une fois que l'héritage nous a trouvés - ou choisis -, l'héritier doit de façon responsable s'in-fidéliser en décidant ce dont il héritera et en l'altérant pour l'étirer, le critiquer, le décodifier ou en hériter de nouveau. Tout ceci, à l'intérieur d'un circuit d'infidélité qui fait de l'héritage un évènement toujours nomade et jamais perturbé par le calcul qu’imprime l'histoire du présent. "Cette réaffirmation qui à la fois continue et interrompt, elle ressemble, au moins, à une élection, à une sélection, à une décision. La sienne comme celle de l'autre : signature contre signature " (Derrida; Roudinesco, 2001, 16).

"Il faudrait penser la vie à partir de l'héritage, et non l'inverse » (Derrida ; Roudinesco, 2001, 16). L'héritage pensé à l'abri de ce principe, c'est-à-dire en tant que générateur de vie et non de mort, est une possibilité pour résister non seulement à l'annihilation d'une pensée ou d'une mémoire philosophique, mais émerge plutôt comme un évènement pouvant préserver la vie en général. Si nous en restons à cette réflexion, il serait possible de penser que l'héritage acquiert chez Derrida une signification transcendantale, et non seulement immanente. Nous voulons dire que c'est uniquement dans le circuit de l'héritage altéré et jamais assumé comme tel qu'il y a un flux, une circulation et des restes, des 
traces, des héritages. L'héritage ainsi compris est en fin de compte un problème insurmontable pour la mort. A ce sujet Derrida écrit : "C'est toujours en réaffirmant l'héritage que l'on peut éviter cette mise à mort » (Derrida ; Roudinesco, 2001, 16).

(Peut-être est-il possible de penser que le legs de Freud continue - aujourd'hui - à être vivant parmi nous en raison du fait qu'il est tombé entre les mains d'héritiers infidèles, d'héritiers qui ont su décoder l'héritage freudien sans obéir aux indications le plus souvent totalisantes de l'institutionnalité psychanalytique ?)

Pour que puisse surgir l'imprédictible, ce qui est sans correspondance, pour que se produise un évènement et pour que la vie soit préservée, il serait nécessaire d'être infidèle à l'héritage avec tout ce qui est à notre disposition. Nous savons déjà que l'héritage vu comme totalité adjugée et préconçue n'est rien de plus qu'un format, une scène inanimée de la délégation qui tendra vers sa propre annihilation. C'est pour cette raison, parce qu'il est vie et circuit de continuité incohérente, que l'héritage tout comme la déconstruction " ne va jamais sans cela, sans amour" (Derrida ; Roudinesco, 2001, 17). Chaque fois que nous faisons quelque chose avec ce dont on a hérité, quelque chose de différent au nom de l'héritage, nous faisons partie d'une distribution de vie et non de mort, de sens inconclus, en arrivant à faire partie d'un devenir émancipé qui clôturerait toute finitude. "Mais rien n'est possible, rien n'a d'intérêt, rien me paraît désirable sans elle (l'héritage). Elle commande deux gestes à la fois : laisser la vie en vie, faire revivre, saluer la vie, "'laisser vivre“" (Derrida ; Roudinesco, 2001, 17).

En prenant en compte tout ce que nous avons analysé jusqu'à présent, il est important de remarquer que l'héritage nous assigne, aux dires de Derrida, des tâches contradictoires. Il ne s'agit pas seulement de recevoir mais aussi de choisir et de réinterpréter, et chacune de ces tâches retombe sur nous et sur notre finitude. La capacité à hériter ne peut être assumée que par un être fini, l'héritage luimême étant néanmoins infini. Lui et les multiples dérivations et interprétations que l'on peut atteindre à partir de la finitude de l'homme. Cependant, c'est précisément cette finitude qui nous oblige et nous engage envers un héritage. Hériter n'est donc pas seulement transformer, choisir, exclure, etc. ce qui nous arrive, ce qui nous est donné depuis un passé, mais plutôt cela implique le geste d'assumer un à-venir de l'héritage qui exerce aussi des pressions pour faire partie du circuit de ce qui peut être hérité en moi. C’est ainsi que je peux être un légataire, d'autres peuvent être les miens, circulation qui exigerait une éthique de la 
responsabilité vis-à-vis de l'héritage, celle-là qui indique qu'il n'y a pas de droit de propriété sur mon legs et que personne ne peut assumer en son nom propre ce dont j'hérite ni encore moins délimiter ce qui peut être hérité en moi.

L'héritage se dissémine alors dans le jeu du passé, du présent et du futur et même dans celui de l'à-venir. Dans cette construction, la temporalité ne se joue pas à propos d'une séquencialité logique, elle n'est pas une chance pour une sorte de corpus textuel et encore moins pour l'essai d'une scène d'écriture logocentrique. Passé, présent, futur et à-venir sont dans et pour l'héritage un champ ouvert de relations hétérogènes et irréductibles qui révèlent et hyperbolisent, dans sa circulation même, l'urgence de la responsabilité. Dans cette même ligne, Derrida signale que « le concept de responsabilité n'a plus le moindre sens hors d'une expérience de l'héritage. Avant même de dire qu'on est responsable de tel héritage " (Derrida; Roudinesco, 2001, 18).

La responsabilité dans l'héritage acquiert une double condition, ou autrement dit, l'héritage exige de nous une double responsabilité liée à une sorte d'anachronie élémentaire. Celle-ci nous indique que nous sommes responsables vis-à-vis d'un passé dont nous héritons mais en même temps d'un à-venir que nous méconnaissons et en face duquel nous devons aussi répondre au nom de l'héritage. L'héritier est doublement endetté en vertu d'un principe de responsabilité. Il y a alors une éthique du déplacement et de l'atemporalité dans l'héritage qui sabote la condition d'un présent en lui. En résumé, l'héritier est responsable envers l'héritage qu'il reçoit depuis un passé et qui laisse disponible pour l'à-venir, mais cette responsabilité ne se joue pas dans le présent de l'assimilation du legs, mais plutôt dans le plus près et le plus loin de l'acte d'hériter. Disons alors qu'il y a héritage malgré l'héritage.

L'héritage malgré l'héritage consiste en l'impossible qui habite le possible. Comme cela a déjà été observé, tout travail d'héritage requiert un déchiffrage de ce dont on hérite, une décodification urgente pour faire de l'héritage même un legs orienté vers l'à-venir. C'est dans cette ligne que tout héritage ne serait rien d'autre que la recherche de ces secrets qui l'imprègnent et le constituent. Lorsque nous parlons d'héritage au sens derridien, nous parlons de secrets qui sont cachés derrière la scène performative de cet héritage. Sur ses arrières, il y a toujours des traces ou des archi-traces, le non-dit ou le vouloir-dire, le non-vu ou le fantasmatique ou, encore, la différenciation hétérogène et exagérée des concepts qui le configurent. Le travail d'héritage est donc un travail de recherche et de décision. Recherche et responsabilité pour pénétrer ce qui fait de l'héritage 
une possibilité toujours ouverte et jamais attachée à l'empire de la métaphysique de la présence. L'héritage est, en ce sens, absence et spectralité. Il est ce qui n'est pas révélé ni dans sa forme ni dans sa disposition performative et, par-dessus tout, il est un espace sans économie possible qui nous invite à nous submerger dans les estuaires multiples de la déconstruction.

Héritage, déconstruction, fidélité pour infidélité, responsabilité, vie, secret. Notions qui viseraient finalement à une grande considération derridienne, à savoir : «(...) nous ne sommes que ce que nous héritons. Notre être est héritage, la langue que nous parlons est héritage " (Derrida, 1996, 34). Nous sommes notre héritage, mais non pas ce dont nous héritons, nous ne sommes pas ce dont nous héritons, mais bien le geste d'hériter. Il n'existerait pas de possibilité pour l'être sans être dans l'héritage. Nous serions, semble-t-il, face à une ontologie de l'héritage mais, insistons-nous, il ne s'agit pas d'une ontologie de l'œuvre héritée, mais bien d'ouvrir la possibilité d'être dans la mesure où on est héritier 5 .

En conséquence, dans cette ligne philosophique, nous ne pouvons pas comprendre l'héritage si ce n'est à l'intérieur de l'espace de la singularité. Nous sommes des héritiers singuliers d'un héritage qui comme tel n'existe pas sinon jusqu’à ce qu'il ait été disloqué, critiqué, désorganisé, et de retour pour se réaffirmer au sein de la dissémination a-téléologique du non-héritable. L'héritier et sa singularité, font de l'héritage un évènement infini en même temps qu'impossible. Nous pouvons penser, avec tous les risques qui y sont attachés, qu'avec l'héritage, nous entrons sur le terrain de l'essence, de l'eidétique et sur celui d'une des analyses derridiennes les plus complexes et les plus hétérogènes, celle de la différance et de son économie fondamentale. Ceci sera cependant l'objet d'un examen en une autre occasion.

A partir de tout ce que nous avons considéré jusqu’à maintenant, qu'est-ce qui nous permettrait d'illuminer ou, au moins, de tenter une représentation

5 Tout en en acceptant le risque, on peut dire que l'héritage se déploierait - ou bien se disséminerait - comme condition ontologique pour l'ontologie de l'être même et, avec cela, Derrida viserait une nouvelle stratégie philosophique de dépassement du dasein heideggérien ou de la métaphysique de la présence en général. Nous n’approfondirons pas cette problématique au long de ce texte, mais il semblait judicieux de pressentir l'héritage comme un des dards derridiens lancés à l'intérieur de la problématique de l'être, de la présence et de la temporalité. En relation à cette problématique, voir par exemple : Derrida, J., Ousia y Grammé. Note sur une note de Sein und Zeit. Première versión publiée in L'endurance de la pensée (livre collectif, Pour Saluer Jean Beaufret), Plon, 1968. Finalement in Marges de la philosophie, Paris, Minuit, 1972 
d'une scène potentielle de l'héritage entre Freud et Derrida? Avant toute tentative d'apporter une réponse à cet impératif sous la forme d'une question, il semble nécessaire de réfléchir sur ce que, pense-t-on, pourraient être les traits singuliers qui nuancent la relation de Derrida avec l'œuvre de Freud. Une relation qui ressemble à un règlement de compte d'un héritage avec Freud mais aussi avec l'institution psychanalytique.

\section{Freud avec Derrida et le principe du plaisir.}

Nous commencerons par un passage d'une lettre que Freud écrivit à Ernest Jones en 1920 et que cite René Major dans son livre Lacan avec Derrida : "Je suis sûr que dans quelques décennies mon nom sera oublié mais que nos découvertes subsisteront" (Major, 2001, 1). S’il est vrai que les sens inconscients qui pourraient finir par construire le signifié et le signifiant de cette phrase, ne pourraient être résolus que par Freud lui-même, on sent glisser sur ces paroles la crainte que la transcendance de l'œuvre, de son œuvre, puisse finir par saboter le nom propre, son nom propre. Le nom de Sigmund Freud, semble-t-il, succomberait face à la puissance de son travail et dépose sa confiance en ce qui, selon son pronostic, est plus que lui-même et son identité, c'est-à-dire, son legs, son héritage. La phrase citée acquiert une force philosophique pour ce qui est le centre de ce travail, c'est-à-dire, la possibilité de penser au legs freudien comme quelque chose de plus qu'un nom propre.

Cependant, il vaut la peine de se poser la question de savoir quel est le nom propre de l'héritage freudien et de quoi nous parlons quand nous évoquons Freud et de son legs. A-t-il été conscient de l'énorme mégastructure, théorique, pratique et institutionnelle qui prendrait corps en son nom ? A-t-il été conscient également du grand dispositif de savoir en lequel arriverait à se convertir l'institution de la psychanalyse? Qu'est-ce que Derrida choisit et altère comme héritage ? Freud ou la psychanalyse?

Au premier abord, nous pensons que Freud s'inscrivait dans une tradition de pensée philosophique proche de Nietzsche ou de Schopenhauer, et qu'il cherchait à s'éloigner d'une sorte d'hégélianisme idéaliste pour se rapprocher de 
l'idéal scientifique de son époque. En ce sens, le pari de Lacan dans la tradition psychanalytique est différent et non de façon partielle, puisqu'il dérive vers une sorte d'anti-scientisme lié davantage à ce l'on pourrait interpréter comme une vision hégélienne du désir et à une " conception heideggérienne de la parole et de la vérité » (Major, 2001, 2). L'important pour ce travail est que c'est précisément à ce moment où se produisait cette sorte de changement de timon et de direction dans la conduite de la psychanalyse que Derrida s'interroge ainsi : «On voudrait nous faire oublier la psychanalyse. Oublierons-nous la psychanalyse ?» (Derrida, 1990). La question, à notre jugement, est d'une énorme importance. Derrida était préoccupé par une sorte de retrait de la psychanalyse freudienne de la discussion philosophique. Il tirait son argument du fait qu’après avoir été le mode de pensée le plus pénétrant des années 60 et 70 - ce même mode qui fut sur le point de disloquer de façon presque définitive le discours philosophique à partir de la logique de l'inconscient - nous assistions à ce moment-là au retrait radical de ce canon d'une manière violente et sans questions ni excuses. Comme si l'héritage de Freud n'avait été rien d'autre qu'un antécédent dans le va-et-vient de positions philosophiques itinérantes. La question de Derrida est, finalement, une inquiétude fondamentale vis-à-vis de l'oubli d'un potentiel héritage de Freud et de son legs. Plus précisément, de Freud ou de son legs ? Derrida s’explique dans cette citation :

Ce qui s'est passé, dans l'air du temps philosophique (...), c'est qu'après un moment d'angoisse intimidée, certains philosophes se sont ressaisis. Et aujourd'hui, dans l'air du temps, on commence à faire comme si de rien n'était, comme si rien ne s'était passé, comme si la prise en compte de l'événement de la psychanalyse, d'une logique de l'inconscient, de " concepts inconscients ", même, n'était plus de rigueur, n'avait même plus sa place dans quelque chose comme une histoire de la raison (...) comme si, dans ce moment de restauration philosophique qui est l'air du temps, car ce qui est à l'ordre du jour, à l'ordre moral de l'ordre du jour, c'est une espèce de restauration honteuse et bâclée (...) comme s'il redevenait légitime, enfin, d'accuser d'obscurité ou d'irrationalisme quiconque complique un peu les choses à s'interroger sur la raison de la raison, sur l'histoire du principe de raison ou sur l'événement, peut-être traumatique, que constitue quelque chose comme la psychanalyse dans le rapport à soi de la raison (Derrida, 1990). 
Si nous nous arrêtons sur cette citation, nous y lisons que Derrida assume la psychanalyse comme aucune autre chose que comme un évènement, c'est-à-dire comme ce qui arrive à partir d'un devenir non planifié et hautement corrosif pour une sorte d'histoire linéale de la philosophie. Cet évènement, qui a menacé la séquencialité de la pensée philosophique au point de presque la déborder, aurait été exposé à cette espèce de restauration honteuse et bâclée de la philosophie et de sa séquence, là où un enjeu quelconque pour décentrer la pensée rationnelle devrait être écarté, enfermé dans un fourre-tout de tailleur et dans l'oubli de la force héréditaire qui la constitue.

Ce qu'il y a derrière ce texte de Derrida, peut-on penser, n'est pas un éloge de la psychanalyse, ni non plus une exagération amoureuse envers son créateur, ce que nous voyons est plutôt un cri presque désespéré pour récupérer l'héritage, pour le choisir malgré la tentative de le faire avorter de la part de la dynamique de l'institutionnalité philosophique. La psychanalyse était déjà des nôtres, elle était née, et cependant la tendance à laisser de côté son legs était précisément abortive. (Peut-on faire avorter ce qui est déjà né ?). Depuis le prisme psychanalytique même, Freud et la psychanalyse apparaissaient comme un traumatisme et une répétition qu'il était impératif d'enfermer dans les souterrains de l'inconscient. Etre justes avec Freud, voilà ce qui était à la base de la prédication derridienne.

René Major écrit ceci: «La psychanalyse, c'est ce que Derrida, lui, n’oublie jamais. Il a avec elle un lien originaire comme avec sa langue maternelle » (Major, 2001, 5). Cependant, tout en assumant cette relation originelle de Derrida avec la psychanalyse, la relation avec la langue maternelle n'en est pas sans tension. Tout ce qui se réfère à une relation avec l'origine est un va-et-vient de déchirures soutenues et vitales qui finissent, certes, par définir ce que nous sommes. Par ailleurs, bien que nous parlions d'une relation imaginaire, l'imaginaire lui-même - quelles qu'en soit la forme ou la figure prise - n'impliquerait pas d'acceptation docile ni le geste de se laisser coloniser sans résister. De plus, on nous dit qu' "il n'y aurait pas eu, il n'y a pas Derrida sans Freud " (Major, 2001, 5). Et de fait, c'est quelque chose que nous acceptons, néanmoins, Derrida ne se conçoit pas ni se laisse concevoir comme une réplique de Freud, ni la déconstruction prétendre être quelque chose comme une fille bâtarde de la psychanalyse ou, dans le meilleur des cas, une fille cadette. S'il n'y a pas de Derrida sans Freud, s'il en 
est ainsi et nous le partageons, ce n'est que dans la mesure où Derrida accueille Freud à partir de la résistance et de la figure de l'héritier infidèle. Il l'assume à partir de l'injonction entre lui-même et son Freud (Bennington, 1996).

Tout compte fait, il est possible de se rendre compte que Derrida trouve en Freud un puissant allié de son intérêt le plus précoce pour déconstruire la pensée logocentrique. S'il est vrai que les concepts derridiens proviennent de la métaphysique, tout d'abord à partir d'une lecture de la trace chez Plotin et plus tard à partir des concepts tirés de l'ontologie heideggérienne,- alors que Freud s'écartait clairement de cette route, comme nous l'avons vu -, des notions telles que Nachträglichkeit (après-coup ${ }^{6}$ ) se répercutent dans l'œuvre derridienne de façon directe. Nachträglichkeit est un concept qui vise la disparition du présent en termes d'expérience spécifique. Le shock d'une expérience traumatique excéderait la temporalité actualisée de son propre déploiement tout en se faisant sentir après. Cette idée de la dilation et de l'espacement vis-à-vis du présent ou de la présence que contient la Nachträglichkeit, est fondamentale pour l'émergence des concepts derridiens tels que trace, archi-trace, différance, restance, spectralité, etc. Derrida s'explique de la façon suivante :

Que le présent en général ne soit pas originaire mais reconstitué, qu'il ne soit pas la forme absolue, pleinement vivante et constituante de l'expérience, qu'il n'y ait pas de pureté du présent vivant, tel est le thème, formidable pour l'histoire de la métaphysique, que Freud nous appelle à penser à travers d'une conceptualité inégale à la chose mémé. (Derrida, 1967, 314).

En conséquence, la critique de la métaphysique de la présence et au présent que nous trouvons chez Derrida (par exemple envers l'ontologie heideggérienne ou la critique littéraire structuraliste ${ }^{7}$ pour ne citer que deux moments) pourrait

${ }^{6}$ Lacan aurait été celui qui donne le sens final à la traduction de l'allemand au français de ce terme freudien. Nachträglichkeit serait alors traduisible par après-coup.

7 On suggère de se référer, par exemple, les critiques que Derrida développe envers la critique littéraire structuraliste, surtout concernant le dénommé "Cercle de Genève » où il vise Jean- 
être héritière d'au moins deux lectures fondamentales, celle de Plotin et de son idée qu'en toute forme on pressent les traces d'une certaine non présence et celle de la Nachträglichkeit freudienne, qui déstabilise et sabote la notion de présent même. Toutes deux effacent le mythe d'une origine dans le présent.

C'est alors que, comme le soutient Derrida, "La possibilité de la trace archivante, cette simple possibilité, ne peut que diviser l'unicité. En séparant l'impression de l'empreinte " (Derrida, 1995, 153). C'est-à-dire que la trace n'est possible que si nous la séparons de ce qui peut être archivé ou de ce qui laisse une marque. Cependant, nous nous trouvons là à nouveau en présence d'une aporie, puisque la trace n'est pressentie - pour le moins - que dans la mesure où ce qui est imprimé a été exécuté. Comment résister à cette injonction de sens que la forme de l'imprimé ou de l'archive cache ? Nous nous immisçons dans ce problème, étant donné que Freud et son legs pourraient être compris comme la trace ou l'absence que l'œuvre derridienne contient, celle qui est imprimée. Tout ceci est contradictoire, la déconstruction refusant d'être une ouvre, bien qu'elle le soit en même temps, celle-ci n'étant possible que dans la mesure où elle est impossible. Nous nous référons à l'œuvre imprimée, historique et avec une temporalité présente. C'est ainsi que Freud serait chez Derrida la marque de l'absence, la présence qui par défaut constitue son œuvre. Tout ceci, à l'intérieur du jeu impossible des traces qui se laissent voir dans l'imprimé. Néanmoins, c'est un jeu de résistances hétérogènes multiples et signifiantes qui sont finalement héritées dans l'œuvre de Jacques Derrida.

Dans cette direction, les lectures de Derrida sur Au-delà du principe $d u$ plaisir rendraient compte de la portée que le legs freudien aurait atteint dans l'élaboration de sa propre philosophie : Comme Derrida en fait un développement presque tout au long de La carte postale, Freud aurait été préoccupé de faire de son héritage quelque chose d'incontestable, d'irréfutable pour ses héritiers (Derrida, 1980, 275-437). Néanmoins, il s'agit plutôt ici de ce qui peut être compris du point de vue de Derrida comme une clause freudienne, l'impossibilité de modifier son testament, comme une possibilité à l'endroit du malgré...C'est-à-dire que s'il est vrai que l'héritage au nom propre (Freud psychanalyse) a pu être protégé contre toute tentative de réinterprétation et de

Pierre Richard et Jean Rousset. cf : Derrida, J., "Force et signification ", in L 'écriture et la differénce, Seuil, Paris, 1967b, pp. 9-49 
critique, ce n'est pas pour un autre motif que celui d'approfondir la motivation des héritiers, l'infidélité par fidélité qui doit - sans rien devoir - caractériser leurs accès et excès resignificatifs envers l'héritage.

Si nous entendons la déconstruction comme un déplacement hétérogène sur une écriture ou une structure formelle, ou comme l'évènement qui exerce des pressions sur ce qui est caché derrière l'impression, ou encore comme l'analyse hyperbolisée d'une œuvre quelconque, nous devons signaler aussi qu'il n'y a pas chez Derrida de rejet à l'égard de Freud mais au contraire un oui à son héritage. Ce oui requiert nécessairement un engagement avec une hyper-analyse de l'œuvre freudienne, étant donné qu'il s’agit d'un oui lié par condition à la responsabilité qu'entraîne le fait d'hériter, c'est un oui au oui, je choisis ton héritage, mais pour le transformer et j'en ferai quelque chose qui n'était pas sans nier ce qu'il était. Freud et Derrida s'unissent et se réunissent dans l'originaire et dans l'indivisible.

Toujours dans la lecture derridienne de $A u$-delà du principe du plaisir, nous trouvons cette phrase de Derrida dans La Carte Postale : "Il s'agissait d'interroger la spécificité (problématique et textuelle) de Au-delà..., de relier l'irréductible d'une "spéculation " à l'économie d'une scène d'écriture, ellemême inséparable d'une scène d'héritage impliquant à la fois Freud et le 'mouvement` psychanalytique " (Derrida, 1980, 313). Freud, que Derrida appelait le spéculateur, aurait élaboré sa théorie sur le Fort-da (Freud, 1992) (loin-ici en français) à partir de l'examen des attitudes d'un enfant de 18 mois - son petitfils - non seulement pour rendre compte de la façon dont le principe du plaisir opérait, mais bien, et dans une large mesure, pour expliquer comment la constitution du langage passe par une expérience de la perte. Dans cette perspective, le Fort-da est une expérience spécifique et totale en même temps dans laquelle se joue la constitution du sujet. Cependant, et au-delà de l'expérience de l'enfant qui jette un objet pour le récupérer ensuite en assumant inconsciemment qu'il s'agit de la figure de sa propre mère, celle qui est là et qui n'y est pas, Derrida voit dans cette scène un geste freudien ayant eu un immense impact, en s'interrogeant en même temps sur la question de savoir comment il est possible que Freud ait pu élaborer toute une œuvre, une scène d'écriture, à partir de la spéculation sur l'expérience d'un enfant qui, de plus, était son petit-fils, c'est-à-dire sur lequel il ne pouvait appliquer aucun autre type d'objectivité. Le déplacement à partir d'un moment d'observation spéculative vers celui de l'élaboration d'une théorie sur Au-delà du principe du plaisir, semble, selon Derrida, profondément significatif pour penser une scène ou une figuration de l'héritage. 
Quand Freud spécule, il choisit, il filtre et décide en actualisant, en faisant des enjeux pour modifier de manière interprétative l'expérience qu'il observe. Il y a dans cette " photographie " de Freud analysant les attitudes de son petit-fils, un principe d'héritage fondamental, celui de recevoir en transformant, sans savoir si les conditions objectives le permettent ou non. C'est dans cette direction que Freud se présenterait aux yeux de Derrida comme un digne infidèle qui assume l'expérience tout juste pour la perturber et l'altérer.

Tout en assumant la rupture textuelle, nous avons cherché à proposer un pari à partir d'un dernier argument qui pourrait rendre compte d'une scène de l'héritage entre Freud et Derrida. Nous sommes partis de la base qu'il n'y a pas de droit de propriété sur l'héritage, ce qui a tendance à valider et à indiquer le chemin vers un autre droit, celui de tout dire. Derrida écrit ceci : "Rien n'a contredit ou de quelque manière contesté l'autorité d'un PP qui revient toujours à lui-même, se modifie, se délègue, se représente sans jamais se quitter " (Derrida, 1980, 314).

En conséquence, rien ne se passera hors de ce principe du plaisir. Tout ce qui se révèle, se déploie, et se distribue depuis et à partir de l'expérience humaine adhère d'une façon ou d'une autre à ce PP qui le mobilise et qui donne le départ à tout. Plus encore, c'est le même PP qui retourne à lui-même. Ce lui-même n'opérerait pas chez Freud plus que comme une sorte d'économie fondamentale, une dilation d'origine qui, cependant, est à l'origine de soi-même. Le PP est une condition du PP. Pour Derrida, celui-ci est le plus au-delà du PP chez Freud, le propre PP du plaisir qui est disséminé comme un espacement fondamental antérieur à toute condensation ontique/ontologique. Il n'y a pas de PP avant le PP, c'est la question. Dans un premier temps, nous nous risquerons à une question: Est-il possible de penser l'interprétation derridienne du PP au centre de l'héritage Freud-Derrida ? Sommes-nous si éloignés de nous risquer à penser Freud comme le PP se disséminant dans la philosophie derridienne ? On peut mettre en jeu le fait de penser que l'héritage de Freud dans l'œuvre derridienne opère en tant que définition que Derrida lui-même ferait du PP, c'est-à-dire qu'il n'y aurait pas, de près ou de loin, de Derrida sans Freud. Chacune des considérations de la déconstruction et des différents moments du travail de Derrida seraient d'une façon ou d'une autre pénétrées par l'héritage freudien. Il n'y aurait pas eu, il n'y a pas Derrida sans Freud, disait René Major, et nous l'appuyons. Bien au-delà du dispositif institutionnel qu'est la psychanalyse et que Derrida a affronté en plus d'une occasion, il n'est pas possible de penser l'émergence de l'œuvre derridienne sans le legs de Freud. Derrida disait : Nous sommes notre héritage, celui que nous 
avons choisi par fidèle infidélité. S’il en est ainsi, Derrida est Freud, mais en lui résistant.

\section{Conclusion}

Ce texte ne prétendait nullement être, comme on a pu le voir, une réflexion technique sur la psychanalyse et ses catégories, encore moins une sorte de trame philosophique qui permettrait l'équivalence de concepts entre la psychanalyse et la déconstruction. Nous sommes tout simplement partis d'une considération critique de Derrida à l'égard de l'institution psychanalytique, celle qui nous dit qu'il n'est pas nécessaire de militer pour la psychanalyse pour s'y référer et qu'une partie importante de l'héritage freudien serait étanche à l'intérieur de l'espace doctrinal que les dénommés psychanalystes professionnels souscrivent.

Pour Derrida, la psychanalyse, particulièrement au niveau institutionnel, a été une science de l'archive et du nom propre. Une institution sélective et spécifique qui approuve ou écarte en opérant presque depuis la même logique de l'inconscient. On archive pour oublier et on relève un nom propre pour se différencier. Néanmoins et par défaut, ce même geste de l'institution produit une altérité, l'anarchive, ce qui résiste à être approuvé ou rejeté en raison d'un dispositif de pouvoir. Autrement dit, tout ce qui est disposé en vue de favoriser une scène de l'héritage. C'est dans cet interstice de sens non pris en considération par l'intentionnalité psychanalytique que ce texte se loge en lui-même, non pas en ses habitacles institutionnels. C'est l'espace que Derrida révèle pour nous qui ne sommes pas membres de la structure formelle de la psychanalyse, et c'est en même temps l'héritage qui stimule.

Nous mettons en jeu le fait de libérer l'héritage freudien de lui-même et de libérer aussi Freud de lui-même en même temps que de ses extensions. Rappelons qu'il n'y a d'héritage que lorsqu'il y a quelque chose d'indicible, que le secret se révèle par excès de forme et de présence. L'héritage est une réponse à l'hétérogène d'un legs et non pas un geste complice et commode vis-à-vis de ce qui a déjà été écrit. L'héritage est multitude de voix et non pas silence face à une archive. Il est, sous la forme d'évènement, la désarchivation. 
Qui hérite ? Freud ou Derrida ? Qui est le légataire final à l'intérieur de cette histoire d'héritages, de passions, d'injonctions, etc. ? Au milieu de tant de trafics d'héritages, de Socrate à Freud, comment situer le grand héritier ?

Nous souhaitons qu'il n'y ait pas de dernier des héritiers et que l'héritage, tout comme la déconstruction, demeure en tant que responsabilité impossible.

\section{Bibliographie}

Bennington, G. (1996). Circanalyse (la chose même), Communication présentée au colloque de Cerisy, Julllet, 1996. Après in Major, R. y Patrick Guyomard (dir), Depuis Lacan (2000). Colloque de Cerisy, Paris : Aubier, Paris, 2000.

De Peretti, C. (2005). "Herencias de Derrida ", Madrid: in Isegoría n³2, pp. 119132

Derrida, J. (1967). "Freud et la scène de l'écriture ", in L'écriture et la differénce, Paris : Seuil

- (1967). «Force et signification », in L'écriture et la differénce, Paris : Seuil

- (1995). Mal d'archive, Paris : Galilée

- (1968). La différance, Conférence prononcée à la Société française de philosophie le 27 janvier 1968, et publiée simultanément dans le Bulletin de la même société (juillet-septembre 1968) et dans Théorie d'ensemble (coll. Tel Quel), Paris : Seuil

- (1972). "Ousia y Grammé. Note sur une note de Sein und Zeit » in Marges de la philosophie, Paris : Minuit

- (1980). "Spéculer-sur- "Freud" ", in La carte postale. De Socrate à Freud et au-delà, Paris : Flammarion

- (1987). Psyché. Inventions de l'autre, Paris : Galilée

- (1990). "Let us not forget - Psychoanalysis », In The Oxford Literary Review, «Psychoanalysis and Literature», $\mathrm{n}^{\text {os }} 1-2$, vol. XII

- (1993). Spectres de Marx. L'État de la dette, le travail du deuil et la nouvelle International, Paris : Galilée

- (1996). Échographies de la télévision. Entretiens filmés, Paris : Galilée-INA,

- (1998). Points de suspension, Paris : Galilée

- (2001). Papier Machine, Paris : Galilée 
Derrida, J. Roudinesco, E. (2001). "Choisir son héritage ", in De quoi demain, Paris : Fayard/Galilée

Freud, S. (1920-1922). Euvres complètes, Traduction de l'allemand de José L. Etcheverry, Volume 18 (1920-22), Bs. Aires : Amorrortu

- (1992). Más allá del principio de placer, Psicología de las masas y análisis del yo y otras obras, Bs. Aires: Amorrortu

Major, R. (2001). Lacan avec Derrida : analyse désistentielle, Paris : Champs Flammarion

LitTré, É. Dictionnaire de la langue française (Version digitale)

Recibido : 24/04/2014

Aceptado : 17/07/2014

Este trabajo se encuentra bajo una licencia de Creative Commons ReconocimientoNoComercial-SinObraDerivada 4.0 
